\title{
Retinocollicular synaptogenesis and synaptic transmission during formation of the visual map in the superior colliculus of the wallaby (Macropus eugenii)
}

\author{
D. L. Flett, ${ }^{\star}$ C. H. Lim, ${ }^{\star}$ S. M. Ho, R. F. Mark and L. R. Marotte \\ Central Nervous System Stability and Degeneration Group, Research School of Biological Sciences, Australian National University, \\ Canberra, ACT 0200, Australia
}

Keywords: activity, electrophysiology, optic, ultrastructure

\begin{abstract}
Spontaneous retinal activity has been implicated in the development of the topographic map in the superior colliculus (SC) but a direct demonstration that it reaches the colliculus is lacking. Here we investigate when the retinocollicular projection is capable of transmitting information from the retina in a marsupial mammal, the wallaby (Macropus eugenii). The projection develops postnatally, allowing in vivo analysis throughout development. Quantification of retinocollicular synaptogenesis has been combined with electrophysiology of the development and characteristics of retinocollicular transmission, including in vivo and in vitro recording in the same animals. Prior to postnatal day $(\mathrm{P})$ 12-14 in vitro recording detected only presynaptic activity in retinal axons in the colliculus, in response to stimulation of the optic nerve. Postsynaptic responses, comprising both $N$-methyl-D-aspartate (NMDA) and non-NMDA responses, were first detected in vitro at P12-14 and retinal synapses were identified. In contrast, postsynaptic responses to optic nerve stimulation could not be detected in vivo until P39, around the time that retinal axons begin arborizing. Around this age density and numbers of total synapses began increasing in the retinorecipient layers of the colliculus. By P55-64, the numbers of retinal synapses had increased significantly and density and numbers of retinal and total synapses continued to increase up to P94-99. During this time the map is undergoing refinement and degenerating axons and synapses were present. The discrepancy between in vitro and in vivo onset of functional connections raises the question of when retinal activity reaches collicular cells in the intact, unanaesthetized animal and this will require investigation.
\end{abstract}

\section{Introduction}

The spatially ordered projection of retinal ganglion cells onto their targets in the mammalian superior colliculus (SC) is widely used as a model system to study the development of neuronal connections, especially the formation of topographic maps. Development of the retinotopic map in the SC has been characterized anatomically in a number of species (Simon \& O'Leary, 1992; Mark et al., 1993; Chalupa et al., 1996; Ding \& Marotte, 1997; Chalupa \& Snider, 1998). The initial retinal projection is only coarsely ordered and a precise map arises primarily through a remodelling process that includes the loss of aberrant branches and refinement of arbors. While recent investigations have concentrated on the molecular cues underlying the formation of such maps (see McLaughlin \& O'Leary, 2005), studies of the formation of eye-specific connections in the brain indicate that neuronal activity arising in the retina might also play a role in the development of the map in the SC (Penn et al., 1998; Huberman et al., 2002; Huberman et al., 2003) but see Cook et al. (1999); Crowley \& Katz $(1999,2000)$. Indeed, pharmacological and genetic manipulations (Simon et al., 1992; Grubb et al., 2003; McLaughlin et al., 2003; Chandrasekaran et al., 2005; Mrsic-Flogel et al., 2005) have

Correspondence: Dr Lauren. R. Marotte, as above.

E-mail: lauren.marotte@anu.edu.au

*D.L.F. and C.H.L. contributed equally to this work.

Received 16 December 2005, revised 8 March 2006, accepted 22 March 2006 implicated spontaneous retinal activity in the process. However, for such activity, either permissive or instructive, to influence the development of the topographic map it must be transmitted to the $\mathrm{SC}$ and direct evidence for this is lacking. Few studies have examined the functional development of the retinocollicular projection especially in placental mammals (Molotchnikoff \& Itaya, 1993; Itaya et al., 1995; Lim \& Ho, 1997) where the initial stages of axonal ingrowth occur prenatally, necessarily limiting such studies to in vitro preparations (Reece \& Lim, 1998). Further, in the rat there is a discrepancy in the reported time of onset of synaptic transmission, which was found to occur prenatally in vitro (Reece \& Lim, 1998) but postnatally in vivo (Lim \& Ho, 1997).

In a marsupial mammal, the wallaby, development of the retinocollicular projection is entirely postnatal. The first retinal axons reach the SC a few days after birth (Ding \& Marotte, 1996) and the stages in forming an ordered topographic map are well described (Mark et al., 1993; Ding \& Marotte, 1997). In this study we have investigated how activity arising in the retina might influence the formation of the topographic map in the SC, by examining the structural and functional development of the retinocollicular projection. Quantitative ultrastructure of retinocollicular synaptogenesis has been combined with an electrophysiological investigation of the onset and characteristics of retinocollicular transmission, including the testing of transmission in both in vivo and in vitro conditions in the same animals. This revealed that although the capacity for retinocol- 
licular transmission is present at early stages of development in the wallaby, it is not detectable in vivo.

\section{Materials and methods}

\section{Animals}

Wallaby pouch young (Macropus eugenii) were obtained from a breeding colony. Experiments were approved by the Animal Ethics Experimentation Committee of the Australian National University. Animals were either of known age (P30-38 and younger) or age was determined from a chart of head measurements of animals of known age $(n=122)$. A straight line regression of age on head length resulted in a table of predicted age as a function of head length with a standard error of 2 days.

\section{Electron microscopy}

Time points chosen were based on developmental milestones in the retinocollicular projection described in previous studies (Mark et al., 1993; Ding \& Marotte, 1996, 1997; Freeman et al., 1997): Postnatal day $(\mathrm{P}) 14(n=3)$ - optic axons cover most of the SC but are still very sparse; P28-30 $(n=4)$ - optic axons are distributed in coarse retinotopic order and are largely unbranched without terminal arbors; P38 $(n=3)$ - immediately prior to the onset of arborization; P42-44 $(n=4)$ - onset of arborization rostrally, concomitant with onset of synaptic activity there in response to optic nerve stimulation in vivo; P59-64 $(n=4)$ - axons from all retinal quadrants have arborized and many aberrant axons have disappeared; P94-99 $(n=3)$ - discrete terminal zones have decreased in size and aberrant axons are absent. Animals aged from P14-28 were anaesthetized by hypothermia while older animals were anaesthetized by intramuscular ketamine (ParkeDavis, $\quad 0.05-2 \mathrm{mg} / 100 \mathrm{~g}$ body weight) and xylazine (0.1$0.2 \mathrm{mg} / 100 \mathrm{~g}$ body weight). The retinal projection to the $\mathrm{SC}$ was labelled by an injection of $5 \%$ horseradish peroxidase conjugated to wheat germ agglutinin (WGA-HRP) in one eye. After 10-28 h survival, to allow for WGA-HRP transport, animals were deeply anaesthetized and perfused briefly with $0.9 \%$ sodium chloride followed by $1 \%$ paraformaldehyde and $2.5 \%$ glutaraldehyde in $0.1 \mathrm{M}$ phosphate buffer (PB) at $\mathrm{pH} 7.4$ for $30 \mathrm{~min}$. The SC was dissected out and kept overnight at $4{ }^{\circ} \mathrm{C}$ in $0.1 \mathrm{M} \mathrm{PB}$. After embedding in $4 \%$ agar, coronal sections through the $\mathrm{SC}$ were cut at a thickness of $100 \mu \mathrm{m}$ with the aid of a vibratome into $0.1 \mathrm{M} \mathrm{PB}$ and reacted to visualize anterogradely transported WGA-HRP according to the cobalt-glucose oxidase diaminobenzidine method (Plummer \& Behan, 1993). For light microscopy, alternate sections were mounted on slides, air-dried overnight, dehydrated and coverslipped. Every fourth section was processed for electron microscopy. Sections of the SC contralateral to the injected eye were divided into medial and lateral samples and postfixed in $1 \%$ osmium tetroxide in $0.1 \mathrm{M} \mathrm{PB}$ at $\mathrm{pH} 7.4$ containing $1.5 \%$ potassium ferrocyanide for $2 \mathrm{~h}$ then dehydrated and embedded in Araldite. Ultrathin sections were cut and stained with lead citrate. Sections were examined in a Hitachi 7100 electron microscope. For the quantitative analysis a series of tracks at right angles to the pial surface were made through the entire depth of the retinorecipient layers of the SC (determined from adjacent sections mounted for light microscopy) at a magnification of 18500 times. Three to four such tracks, of $5.9 \mu \mathrm{m}$ in width, were made for both medial and lateral samples taken from the rostral one quarter of the SC in each animal. Every terminal making a synapse was photographed, the criterion for a synapse being a minimum of three synaptic vesicles adjacent to a postsynaptic membrane with a clear specialization. The synaptic contact length of both unlabelled synaptic terminals and those containing WGA-HRP reaction product was measured at each age. For animals of age P14-P38 all synaptic contact lengths were measured. For older animals a minimum of 24-31 from each medial and each lateral sample were measured. The density of all labelled and unlabelled synapses was calculated. Mean numbers of both types of synapses per track through the retinorecipient layers were also calculated to take into account the increase in thickness of these layers during development. The total area of tissue examined in each animal ranged from 4307 to $7434 \mu^{2}$. Control tissue came from two animals, aged P90 and P100, which did not receive intravitreal injections of WGA-HRP but in which the tissue was processed as described above.

\section{Electrophysiology}

For in vitro studies of whole collicular preparations 39 pouch young, aged from P4 to P36 were anaesthetized by hypothermia and decapitated. The head was quickly transferred to a dissecting bath superfused with chilled (below $10^{\circ} \mathrm{C}$ ) isotonic sucrose solution with the following composition: sucrose, $266 \mathrm{mM} ; \mathrm{KCl}, 3 \mathrm{mM} ; \mathrm{CaCl}_{2}$, $2 \mathrm{mM} ; \mathrm{MgCl}_{2}, 1 \mathrm{~mm} ; \mathrm{NaHCO}_{3}, 17 \mathrm{mM}$; D-glucose, $12 \mathrm{~mm}$; saturated with $95 \% \mathrm{O}_{2}, 5 \% \mathrm{CO}_{2}$. After immersion a craniotomy was carried out to expose the brain. The brain was then gently lifted to reveal the optic nerves, which were cut as close to the eye as possible. The brains were split longitudinally along the midline except at the optic chiasma, where both optic nerves were left attached to the side used for recording. The cerebral cortex was removed. After the dissection and a rest period of approximately $1 \mathrm{~h}$, the preparation was transferred to the recording chamber. The preparation was laid on its medial side and secured with nylon threads onto the Sylgard base of the chamber. The chamber was superfused with artificial cerebrospinal fluid (ACSF) with the following composition: $\mathrm{NaCl}, 124 \mathrm{mM} ; \mathrm{KCl}, 5 \mathrm{mM} ; \mathrm{CaCl}_{2}$, $2 \mathrm{mM} ; \mathrm{MgSO}_{4}, 2 \mathrm{mM}$; $\mathrm{NaHCO}_{3}, 26 \mathrm{mM}$; $\mathrm{NaH}_{2} \mathrm{PO}_{4}, 1.25 \mathrm{~mm}$; D-glucose, $11 \mathrm{~mm}$; saturated with $95 \% \mathrm{O}_{2}$ and $5 \% \mathrm{CO}_{2}$, and bath temperature was maintained at $29{ }^{\circ} \mathrm{C}$.

For in vitro collicular slices ten pouch young, aged from P40 to P142 were anaesthetized as above, both colliculi were removed and placed in chilled ASCF saturated with $95 \% \mathrm{O}_{2}$ and $5 \% \mathrm{CO}_{2}$, trimmed and mounted for sectioning. Sagittal slices, $400-\mu \mathrm{m}$ thick, were cut on a vibratome in chilled isotonic sucrose solution saturated with $95 \% \mathrm{O}_{2}$ and $5 \% \mathrm{CO}_{2}$. Prior to recording they were transferred to ASCF saturated with $95 \% \mathrm{O}_{2}$ and $5 \% \mathrm{CO}_{2}$ at $37{ }^{\circ} \mathrm{C}$ for $30 \mathrm{~min}$ and then held at room temperature for at least $60 \mathrm{~min}$. Recording was at $30{ }^{\circ} \mathrm{C}$.

In vivo experiments were performed on six pouch young wallabies of either sex, aged from P25 to P49. The pouch young were anaesthetized with intraperitoneal injection of ethyl carbamate (Urethane, Fluka AG, 20\% w/v) at a dose of $3 \mathrm{mg} / 100 \mathrm{~g}$ body weight. Additional anaesthesia was given 2-4 times at one-quarter of the original dose every half hour and thereafter when needed. The animal was placed in an artificial pouch consisting of a flexible polythene bag surrounded by a water jacket kept at $37^{\circ} \mathrm{C}$. The head, held in a holder mounted on a ball joint, was fixed in a standard position with the palprebal fissure horizontal, as measured by a sighting spirit level. The electrocardiogram was recorded continuously and monitored on a chart recorder and loudspeaker. This served as an indicator of the physiological condition and level of anaesthesia of the animal. A drill hole approximately $1 \mathrm{~mm}$ in diameter was made in the skull directly above the SC. The dura was pierced and the recording electrode was advanced by a piezoelectric stepper (Burleigh Instruments) so angled that the electrode penetrated the colliculus perpen- 
dicular to the collicular surface. At the end of the in vivo experiment whole collicular preparations from each animal were set up for in vitro recordings as described above.

Recording electrodes for both in vitro and in vivo experiments were made of glass capillary filled with either $2 \mathrm{M}$ sodium acetate or $1 \mathrm{M}$ sodium chloride and had a resistance of $1 \mathrm{M} \Omega$ or less. Optic nerve stimulation (whole colliculus preparations) or optic tract stimulation (collicular slice preparations) was delivered by a glass coated bipolar tungsten electrode. Square pulses $(0.02-0.2 \mathrm{~ms}$ duration, $1-80 \mathrm{~V})$ were used at a frequency of $1-60 / \mathrm{s}$ for in vitro experiments. For the in vivo study, the eyelid and cornea were cut horizontally and the lens and most of the vitreous humour removed. The stimulating electrode was placed on the contralateral optic nerve head under visual guidance. The optic nerve was stimulated with square pulses using similar parameters to those used in the in vitro experiments. The stimulus strength was kept below that which would produce any movement of the eye or circumorbital tissues. Optic nerve-evoked potentials recorded in the superior colliculus were amplified using a Neurolog AC amplifier (Neomedix Systems) and band pass filtered at 0.2-2000 Hz for in vivo experiments. An Axoclamp amplifier (Axon Instruments) was used for in vitro preparations and the signal was low pass filtered (DC to $2000 \mathrm{~Hz}$ ). The signal was digitized at $10 \mathrm{kHz}$, averaged and stored using a personal computer. A calibration pulse of $100 \mu \mathrm{V}$ was sometimes given before the nerve stimulus.

Pharmacological agents used during in vitro recording, bicuculline methiodide (+/-)-2-amino-5-phosphovaleric acid (APV) and 6,7dinitroquinoxaline-2,3-dione (DNQX), were all purchased from Research Biochemicals International. They were prepared as 1000× stock solutions and added directly to the ACSF. DNQX was prepared using dimethylsulfoxide (DMSO). In two control experiments the same concentration of DMSO added to the bath had no effect on the optic nerve-evoked potential recorded in the colliculus. For recording of activity in the optic nerve in response to stimulation in the SC a tight fitting suction electrode was used.

\section{Results}

\section{Anatomy}

HRP reaction product appeared as small electron dense profiles of variable shape in axons and axon terminals (Plummer \& Behan, 1993; Fig. 1B-E; see also Fig. S1 in Supplementary material). They were not seen in structures that could be identified as dendrites, cell bodies or glia, suggesting that there was no transneuronal transport of WGAHRP in this situation. Such profiles were absent in controls that did not receive an intravitreal injection of WGA-HRP prior to histochemical processing.

The mean synaptic contact length of synapses in medial and lateral samples, both labelled and unlabelled, did not differ significantly at each age group (two-sided, unpaired $t$-test, data not shown), meaning that synaptic densities and numbers could be validly compared between these samples at each age. The only significant difference found was in unlabelled synapses at P29. Density (numbers $/ 100 \mu \mathrm{m}^{2}$ ) was significantly higher $(P=0.003)$ laterally $(1.15 \pm 0.11)$ than medially $(0.47 \pm 0.09)$ as was the number of synapses/column (14.41 \pm 1.41 laterally, $6.36 \pm 1.21$ medially; $P=0.005)$. Results from medial and lateral samples have been combined in Fig. 2. Mean synaptic contact lengths of labelled and unlabelled synapses did not differ significantly over age (ANOVA, data not shown), allowing densities and numbers to be validly compared. Inputs from interneurons, deeper collicular layers and brainstem may contribute to unlabelled synapses.
At P14 the superficial retinorecipient region of the SC had large extracellular spaces (Fig. 1A), a feature of immature SC (Cooper \& Rakic, 1983; Warton \& McCart, 1989; Correa-Gillieron \& Cavalcante, 1999). Terminals making synaptic contact were extremely rare, especially labelled terminals (Fig. 1B). Synapses often appeared to be made between axonal growth cones of irregular shape containing flocculent material (Fig. 1A) and dendritic growth cones with similar characteristics (Skoff \& Hamburger, 1974) (Fig. 1C). Labelled axons, sometimes containing vesicles, were seen more frequently (Fig. 1A). At P28-30 and P38 extracellular space remained prominent and bundles of unmyelinated axons, some containing reaction product, increased in the superficial layers (Fig. 1D) but synapses remained low in number and density (Fig. 2). A labelled growth cone just beneath the pial surface is seen in Fig. 1D making a synapse behind the tip. Rare labelled axosomatic synapses were also seen (Fig. 1E). At P4244 there was a significant increase in total numbers of synapses compared to the previous age (Fig. 2D; ANOVA, $P=0.02$ ) although labelled synapses (Supplementary material, Fig. S1, A) showed no significant changes (Fig. 2A and B). Extracellular space was also reduced. The most striking feature of the neuropil at P55-64 was the presence of numerous degenerating profiles, including unlabelled (Fig. 1F) and labelled (Fig. 1G) synapses and axons (Fig. 1H). Despite this there were significant increases in both density (Fig. 2C, ANOVA, $P=0.03$ ) and numbers (Fig. 2D, ANOvA, $P=0.005$ ) of total synapses over that seen at P42-44. While labelled synapses had increased (Fig. 2A and B), this was not significantly different compared to the P42-44 data. However, a significant increase in numbers of labelled synapses was detected over the period from P14 to P55-64 (Fig. 2B, ANOVA, $P=0.04$ ). By P94-99 there were further large increases in the density (Fig. 2A and C) and numbers (Fig. 2B and D) of labelled and total synapses (ANOVA; labelled synapses, density $P=0.02$; numbers $P=0.003$; total synapses, density $P=0.004$; numbers $P=0.003$ ). Overall, the increases in these measures over the period P29 and P94-99 were extremely significant (ANOVA, $P<0.0001$ ). Degeneration was still prominent at P94-99, and included neuronal profiles containing vesicles and synapses and dendrites (Supplementary material, Fig S1, B and C, respectively). Extracellular space had decreased further.

\section{Electrophysiology - in vitro recording}

A diagram of the in vitro preparation is shown in Fig. 3A. At P4 an optic nerve-evoked potential of small amplitude $(20-80 \mu \mathrm{V})$ was detected only in the superficial layer of the rostrolateral region of the SC (Fig. 3A, position a). The response attenuated more caudally (Fig. 3A, position $\mathrm{b}$ ) and no response was observed beyond onequarter of the rostrocaudal extent of the colliculus (Fig. 3A, position c). This is consistent with a previous histological study (Ding \& Marotte, 1996), showing that retinal afferents have just arrived in the rostrolateral region of the $\mathrm{SC}$ at this age. At $\mathrm{P} 9$ in the rostral $\mathrm{SC}$ (Fig. 3B) a response characterized by two negative peaks was observed when the stimulation frequency was kept below $0.03 \mathrm{~Hz}$. At approximately $1 \mathrm{~Hz}$ this waveform changed to a single peak response with a reduction in amplitude (Fig. 3B, last trace), suggesting that some axons are not capable of following the higher stimulation rates at this age. The rate used in all subsequent experiments was standardized to once every $30 \mathrm{~s}(0.03 \mathrm{~Hz})$. At P4-9 the response attenuated when the recording electrode penetrated into the deeper layers of the colliculus and no reversal of the waveform was observed. Bath application of glutamate antagonists had no effect on the response (data not shown). These findings indicate that the responses recorded 

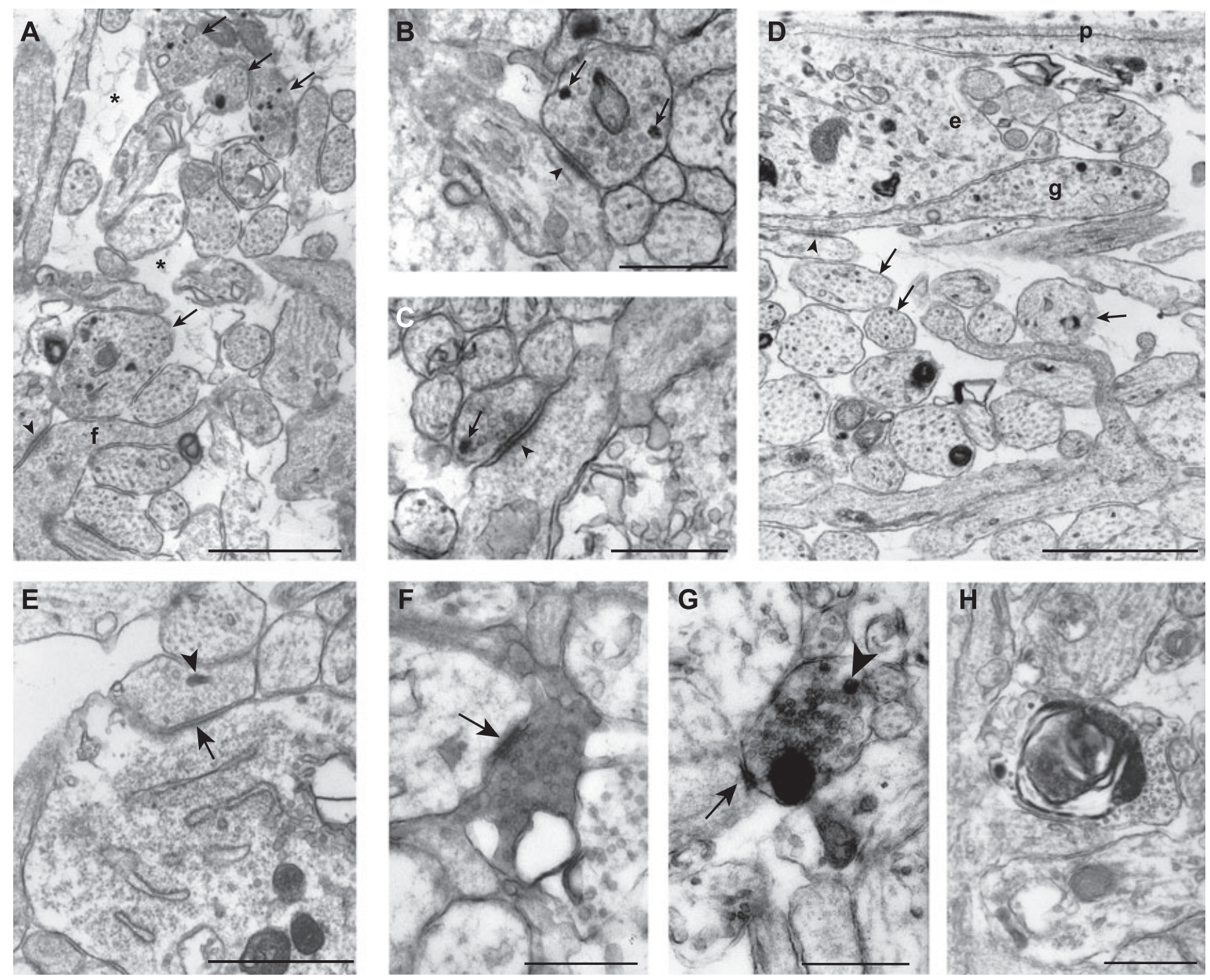

FIG. 1. (A) Low power view within the retinorecipient layers of the superior colliculus in an animal on postnatal day 14. Extracellular space is prominent (asterisks) and few synapses are present (arrowhead). The axon terminal of the one shown contains floccular material (f) characteristic of a growth cone. Numerous unmyelinated axons are present. Retinal axons (arrows), including preterminal axons containing vesicles, can be identified by the presence of electron dense reaction product. Scale bar, $1 \mu \mathrm{m}$. (B and C) Examples of labelled retinal synapses on P14. Arrows indicate the electron dense reaction product and arrowheads indicate the synaptic contact. The postsynaptic profile in C contains floccular material characteristic of a dendritic growth cone. Scale bars, $0.5 \mu \mathrm{m}$. (D) Low power view at the pial surface (p) in an animal on P38. Numerous unmyelinated axons are present. Some can be identified as retinal (arrows) by the presence of electron dense reaction product. An arrowhead indicates a putative synapse made by an retinal axon proximal to its growth cone (g). e, glial endfoot. Scale bar, $1 \mu \mathrm{m}$. (E) Axosomatic synapse (arrow) made by a retinal terminal in the retinorecipient layer of the SC in an animal on P38. Electron-dense reaction product in the terminal is indicated by an arrowhead. Scale bar, $1 \mu \mathrm{m}$. (F-H) Degenerating profiles in the retinorecipient layers of the SC in an animal on P64. (F) Degenerating synapse characterized by increased electron density in the axon terminal. The arrow indicates the synaptic contact. (G) Degenerating retinal synapse (arrow), identified by horseradish peroxidase reaction product (arrowhead), containing electron dense debris. (H) Degenerating axon. Scale bars, $0.5 \mu \mathrm{m}$.

in the more superficial layers of the SC originated from presynaptic volleys in optic axons.

At P12-14 a negative going potential could be recorded at the surface in the rostrolateral half of the SC in response to optic nerve stimulation and it extended to the rostral three-quarters by P20. This mirrors the pattern of ingrowth of retinal axons (Ding \& Marotte, 1997). Within the rostral quarter of the SC the waveform of the evoked potentials was more complex than seen previously. The response changed from a negative wave on the surface to a positive wave of slightly longer latency at $400 \mu \mathrm{m}$ (Supplementary material, Fig. S2), indicating that it is arising from the synaptic current of postsynaptic cells (Freeman et al., 1997). To confirm that the response included a postsynaptic component, glutamate blockers were used in two preparations from animals aged $\mathrm{P} 12$ and P14 (Fig. 3C and D). Removal of bath $\mathrm{Mg}^{++}$revealed a slow potential (Fig. 3C, second trace) which could be blocked by the addition of APV, a $N$-methyl-Daspartate (NMDA) channel antagonist (Fig. 3C, third trace). The fast component (arrow in Fig. 3C) was blocked by the non-NMDA glutamate blocker, DNQX (Fig. 3C, fourth trace). The remaining waveform was presumably generated by presynaptic volleys in optic axons. DNQX had no effect on axonal conduction along the optic nerve (Fig. 3D). These findings suggest that optic axons have formed functional synapses in the superficial layers of the rostral SC as early as $\mathrm{P} 12-14$. 

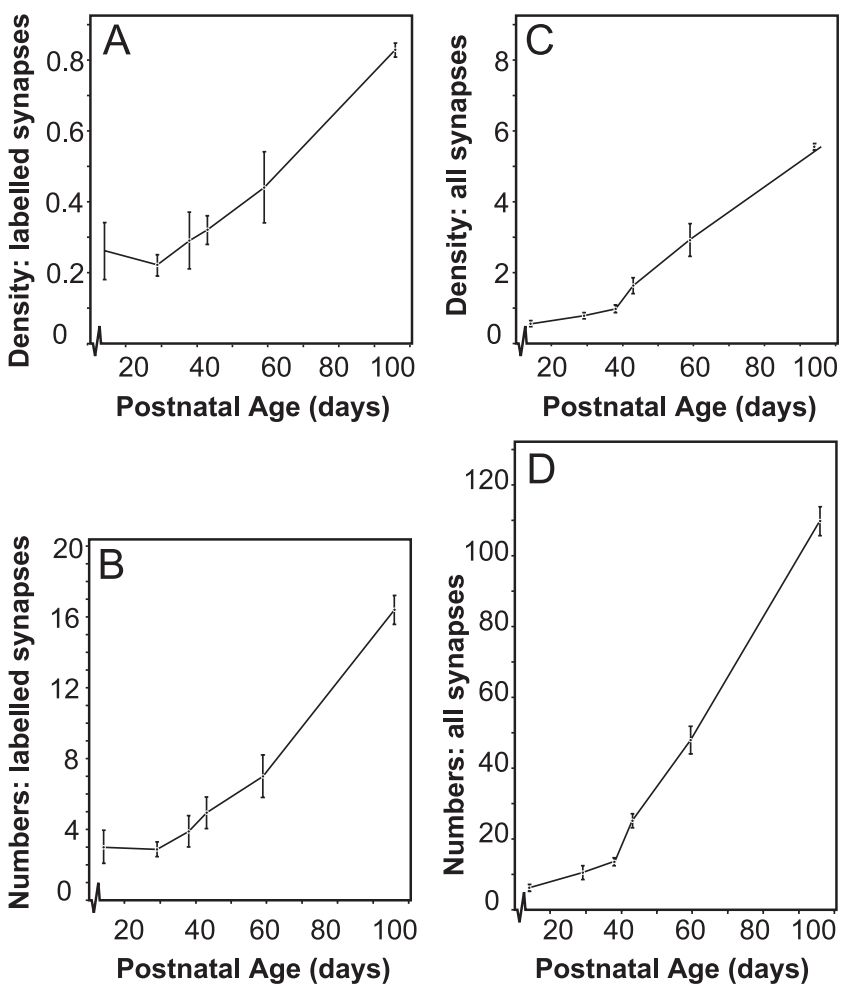

FIG. 2. (A) Mean density (number/100 $\mu \mathrm{m}^{2}$ ) of labelled, retinal synapses in the $\mathrm{SC}$ at different ages. (B) Mean numbers of labelled, retinal synapses calculated from counts in tracks 5.9- $\mu \mathrm{m}$ wide through the retinorecipient layers of the SC at different ages. (C) Mean density (number/100 $\mu^{2}$ ) of all synapses in the retinorecipient layers of the SC at different ages. (D) Mean numbers of all synapses calculated from counts in tracks $5.9-\mu \mathrm{m}$ wide through the retinorecipient layers of the SC at different ages. Error bars show the standard error of the mean.

By P26-36 $(n=3)$ an evoked response, reversing with depth, could be recorded from the entire SC and glutamate blockers had a similar effect to that described at P12-14. Thus by P26 optic nerve axons are capable of mediating synaptic activity across the entire SC.

Although a detailed study was not made in older animals, both NMDA (responses blocked by APV) and non-NMDA responses (blocked by DNQX) contributed to the glutamatergic postsynaptic responses at P59-60 and at P140-142, just after eye opening. In the latter age group non-NMDA responses dominated the response.

\section{Comparison of in vitro and in vivo recording}

Current source density analysis of evoked potentials in response to optic nerve stimulation in vivo did not detect synaptic activity until P45 (Freeman et al., 1997), in contrast to our present results in vitro, where it was demonstrated at $\mathrm{P} 12-14$. To test for in vitro vs. in vivo differences and to minimize the variability between animals, in vivo recordings were made in the SC in six animals aged P25 to P49, followed by in vitro recordings in the same animals. In four animals aged younger than P39, there was no reversal of the polarity of the optic nerve-evoked response recorded in vivo. A negative-going waveform was recorded at the surface and this attenuated when the electrode was advanced deeper into the SC, indicating presynaptic activity only. However, a clear polarity reversal appeared in vitro. Figure 4 illustrates responses in an animal aged P32. Only a negative waveform was recorded in vivo, while in vitro the
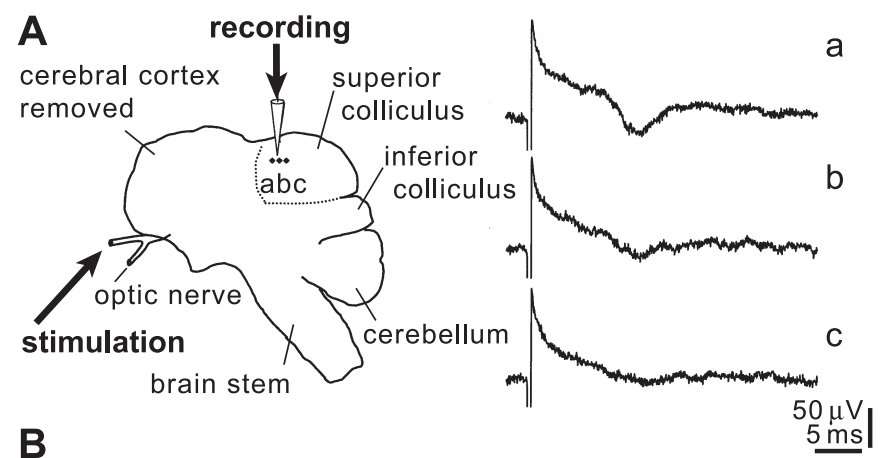

B
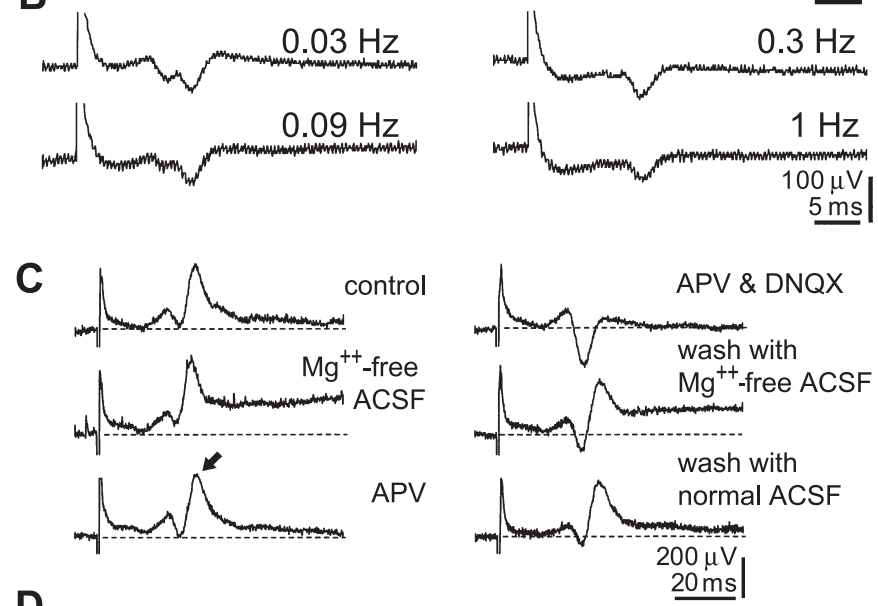

D

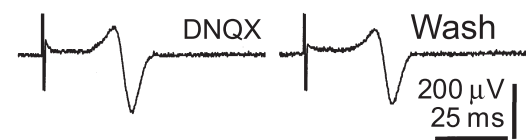

FIG. 3. (A) Left hand panel shows a diagram of a lateral view of the in vitro preparation. Rostral is to the left. The recordings obtained from positions $\mathrm{a}, \mathrm{b}$ and $\mathrm{c}$ in the colliculus of an animal on P4, after stimulation of the optic nerve are shown in the corresponding panels on the right. As the recording electrode was moved caudally the response decreased (b) and then disappeared (c). (B) In vitro recordings from the rostral colliculus of an animal on P9 in response to stimulation of the optic nerve at different frequencies of stimulation. With increasing frequency the waveform changed to a single peak and attenuated. (C) Effects of APV and DNQX on the response in the rostral quarter of the colliculus to stimulation of the optic nerve in an in vitro preparation from an animal at P14. Each trace is an average of ten sweeps at a frequency of stimulation of $0.03 \mathrm{~Hz}$. Apart from the responses shown in the first and last traces $10 \mu \mathrm{M}$ bicuculline was present in the ACSF to block any local inhibition. The control trace shows the response in ACSF at a depth of $300 \mu \mathrm{m}$ from the surface. In magnesium-free ACSF (second trace) a slow potential was present, which was blocked by $50 \mu \mathrm{M}$ APV (third trace). The fast component of the response (arrow) was unaffected but was blocked by the addition of $2 \mu \mathrm{M}$ DNQX (fourth trace). The residual response presumably represents the presynaptic volley in optic axons (see D). Responses returned to control levels in magnesium-free ACSF (fifth trace) and normal ACSF (sixth trace). (D) Control experiment showing that DNQX had no effect on optic nerve conduction. The control trace shows the response in the optic nerve to stimulation in the colliculus. With the addition of DNQX $(2 \mu \mathrm{M})$ there was no change in the response.

waveform reversed in polarity in the deeper layers of the SC, indicating functional retinocollicular transmission. Addition of $20 \%$ urethane in vitro at this age, approximately 100 times the dose in vivo, did not block synaptic transmission (Supplementary material, Fig. S3). This argues against a direct effect of anaesthetic on retinocollicular transmission in vivo. In the remaining two animals aged P39 and P49 the polarity of the response reversed with depth in vivo, indicating postsynaptic activity. Under in vitro conditions, a similar reversal was 


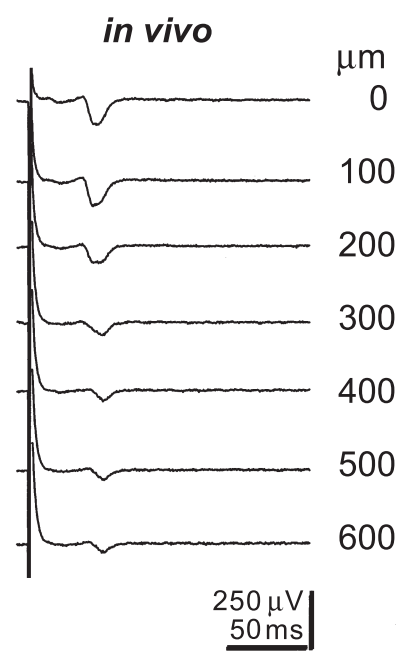

FIG. 4. Responses at different depths in the colliculus to stimulation of the optic nerve in an animal on P32. Each trace is an average of five sweeps at a frequency of stimulation of $0.03 \mathrm{~Hz}$. Responses recorded in vivo show a nonreversing wave form with depth indicative of presynaptic activity only, representing the response of optic axons. In vitro there was a clear reversal of the potential with depth, indicative of a postsynaptic response.

observed between 600 and $750 \mu \mathrm{m}$ below the surface of the SC, close to the recording site in vivo (data not shown).

\section{Discussion}

This study in the wallaby has shown that optic axons, in vitro, are capable of conducting impulses from the time they reach the SC. Postsynaptic responses first appear in vitro at P12-14 and optic synapses are present at this time. However, in vivo postsynaptic responses are first evident much later, at P39, about the time that synapses begin to increase in number in the retinorecipient layers of the SC. By P55-64 identified optic synapses have increased significantly and continue to increase up to P94-99, during the period when the retinocollicular map is refining and degenerating profiles are common.

\section{Development of retinal synapses and map formation}

In the initial stages of formation of the retinotopic map in the $\mathrm{SC}$, largely unbranched retinal axons, tipped with growth cones are distributed in coarse retinotopic order (Ding \& Marotte, 1997). The present study shows that these axons, including growth cones, are already making synapses. In contrast, in the mouse (Sachs et al., 1986) synapses were not detected prior to axonal arborization. In the wallaby axons begin arborizing at $\mathrm{P} 41-45$ and in their retinotopically correct positions (Ding \& Marotte, 1997). Both retinal and total synaptic density and numbers increase from around this time up to the oldest age examined. Concomitant with this, the topographic map is undergoing refinement with elimination of aberrant axons and a decrease in size of terminal zones (Ding \& Marotte, 1997). Refinement is achieved, at least in part, by degeneration of both axons and axon terminals as shown by their presence in the SC during this time. The degeneration coincides with the death of retinal ganglion cells (Spira \& Marotte, 1989).

\section{NMDA and non-NMDA components comprise the retinocollicular response}

The glutamatergic excitatory response comprised both NMDA and non-NMDA components from its onset. These components have also been described in the retinocollicular pathway of the rat at the time of onset of synaptic transmission (Reece \& Lim, 1998) and also later in development (Isa et al., 1998). They are also a feature of glutamatergic transmission in early postnatal mouse colliculus (Juttner et al., 2001). NMDA receptors are involved in activity-dependent refinement of developing projections (Constantine-Paton et al., 1990) and antagonists disrupt the removal of aberrant retinal axons in the rat colliculus (Simon et al., 1992). In the wallaby they have the potential to mediate activity-dependent effects both during the early stage of coarse retinotopy and during the refinement of the projection after terminal zones form. However, any effect of retinal activity during the early stage may be precluded, as retinocollicular synapses may not be transmitting neural activity in vivo (see below). It is possible that the synapses may be mediating trophic effects at this stage. For example, brain-derived neurotrophic factor is expressed in the visual system of the wallaby (Marotte et al., 2004), as it is in other species and it has multiple roles in visual development (Cohen-Cory \& Lom, 2004).

\section{Onset of transmission in vivo is later than in vitro}

There was a difference of approximately a month between the onset of retinocollicular transmission, detected in vitro (P12) and in vivo (P39). The onset of transmission in vivo is around the time that retinal axons begin arborizing in the SC (Mark et al., 1993; Ding \& Marotte, 1997) and is well before the possibility of light-evoked responses. In the wallaby photoreceptor outer segments do not begin to form until P85 (Spira \& Marotte, 1989) and eye opening is around P140. Furthermore, we find that the onset in vivo is not accompanied by either the initiation of, or a significant increase in retinocollicular synaptogenesis. In the rat SC a similar difference between in vitro (at E20; Reece \& Lim, 1998) and in vivo (at P2; Lim \& Ho, 1997) onsets has been reported and in both the rat (Simon \& O'Leary, 1992) and wallaby the in vivo onset is close to the time that axons begin arborizing in the SC.

There are a number of possible reasons for the difference in onset between the two conditions. Anaesthetic and temperature would be likely candidates but the anaesthetic used in vivo did not suppress synaptic transmission in vitro and the lower temperature in vitro compared to in vivo would be more likely to detrimentally affect rather than enhance potentials. We consider firstly, that the in vitro preparation may not reflect the real in vivo physiology. In vitro, inputs to the SC have been removed or disrupted, which may normally inhibit retinal responses, although cortical input is not one. Cortical axons do not reach the SC until P71 (Sheng et al., 1990) and are not functional until around eye opening (Freeman et al., 1997). Secondly, an increase of up to $60 \%$ in synapse numbers has been reported in slice preparations compared to perfusion fixed hippocampus (Kirov et al., 1999). The suggestion that this is in response to the loss of synaptic activity when the slices are first removed from the rest of the brain is supported by experiments in hippocampal slices in which transmission was blocked (Petrak et al., 2005). If a similar increase in synaptogenesis occurred in the present experiments in vitro this may have increased postsynaptic responses to a detectable level. Thirdly, a suppressive mechanism may exist in vivo to inhibit the onset of electrical activity. There is a frequency-dependent gamma-aminobutyric acid (GABA) mediated attenuation of conduction in the optic nerve of the developing rat (Sakatani et al., 1991; Lim \& Ho, 1998) and wallaby (Lim, 1997). This may act to prevent the transmission of excessive activity from the retina in the early postnatal period when local inhibitory mechanisms are poorly developed in the SC (Warton et al., 1990; Shi et al., 1997). In vitro, GABA may leach out and improve conduction in the optic nerve (Reece \& Lim, 1998). 


\section{Implications for the role of activity in formation of the map}

A number of recent studies have emphasized a role for spontaneous retinal activity in the development of coarse retinotopy in the SC (Mrsic-Flogel et al., 2005) and in the refinement of the map in the lateral geniculate nucleus (Grubb et al., 2003) and the SC (Chandrasekaran et al., 2005). However, the only study to demonstrate that such activity reaches the visual centre under investigation was an in vitro study of the retinogeniculate pathway in the mouse (Mooney et al., 1996). The present study shows that activity arising in the retina has the potential to influence the formation of the topographic map over almost its entire development but the later onset of synaptic transmission in vivo suggests that it is only during the period of arborization and refinement that activity may reach the SC. We also observed attenuation of optic nerve responses at frequencies considerably less than those that spontaneous retinal activity can reach (Galli \& Maffei, 1988). Further, spontaneous activity in the rat SC during refinement of the projection was unaffected by blocking transmission in the optic nerve (Itaya et al., 1995). All these findings indicate that investigation of the developing retinocollicular pathway in an unanaesthetized, intact animal will be necessary to determine exactly when retinal activity reaches the SC. This would be feasible in the wallaby, with its protracted postnatal development of the retinocollicular pathway.

\section{Supplementary material}

The following supplementary material may be found on http://www.blackwell-synergy.com

Fig. S1. Retinal synapse and degenerating profiles in the SC.

Fig. S2. Evoked responses in the SC in vitro on P12.

Fig. S3. Effect of urethane on the evoked response in the SC in vitro on $\mathrm{P} 32$.

\section{Acknowledgements}

We thank Dr C. A. Leamey and Dr J. Provis for criticism of the manuscript, Dr J. Hemmi for statistical advice, Mr K. Williams and Ms M. Maier for animal care and Ms S. Wragg for preparation of the figures.

\section{Abbreviations}

ACSF, artificial cerebrospinal fluid; APV, (+/-)-2-amino-5-phosphovaleric acid; DNQX, 6,7-dinitroquinoxaline-2,3-dione; NMDA, $N$-methyl-D-aspartate; $\mathrm{P}$, postnatal day; PB, phosphate buffer; SC, superior colliculus; WGA-HRP, horseradish peroxidase conjugated to wheat germ agglutinin.

\section{References}

Chalupa, L.M. \& Snider, C.J. (1998) Topographic specificity in the retinocollicular projection of the developing ferret: an anterograde tracing study. J. Comp. Neurol., 392, 35-47.

Chalupa, L.M., Snider, C.J. \& Kirby, M.A. (1996) Topographic organization in the retinocollicular pathway of the fetal cat demonstrated by retrograde labeling of ganglion cells. J. Comp. Neurol., 368, 295-303.

Chandrasekaran, A.R., Plas, D.T., Gonzalez, E. \& Crair, M.C. (2005) Evidence for an instructive role of retinal activity in retinotopic map refinement in the superior colliculus of the mouse. J. Neurosci., 25, 6929-6938.

Cohen-Cory, S. \& Lom, B. (2004) Neurotrophic regulation of retinal ganglion cell synaptic connectivity: from axons and dendrites to synapses. Int. J. Dev. Biol., 48, 947-956.

Constantine-Paton, M., Cline, H.T. \& Debski, E. (1990) Patterned activity, synaptic convergence, and the NMDA receptor in developing visual pathways. Annu. Rev. Neurosci., 13, 129-154.

Cook, P.M., Prusky, G. \& Ramoa, A.S. (1999) The role of spontaneous retinal activity before eye opening in the maturation of form and function in the retinogeniculate pathway of the ferret. Vis. Neurosci., 16, 491-501.
Cooper, M.L. \& Rakic, P. (1983) Gradients of cellular maturation and synaptogenesis in the superior colliculus of the fetal rhesus monkey. J. Comp. Neurol., 215, 165-186.

Correa-Gillieron, E.M. \& Cavalcante, L.A. (1999) Synaptogenesis in retinoreceptive layers of the superior colliculus of the opossum Didelphis marsupialis. Brain Behav. Evol., 54, 71-84.

Crowley, J.C. \& Katz, L.C. (1999) Development of ocular dominance columns in the absence of retinal input. Nature Neurosci., 2, 1125-1130.

Crowley, J.C. \& Katz, L.C. (2000) Early development of ocular dominance columns. Science, 290, 1321-1324.

Ding, Y. \& Marotte, L.R. (1996) The initial stages of development of the retinocollicular projection in the wallaby (Macropus eugenii): distribution of ganglion cells in the retina and their axons in the superior colliculus. Anat. Embryol. (Berl.), 194, 301-317.

Ding, Y. \& Marotte, L.R. (1997) Retinotopic order in the optic nerve and superior colliculus during development of the retinocollicular projection in the wallaby (Macropus eugenii). Anat. Embryol. (Berl.), 196, 141-158.

Freeman, T.C., James, A.C. \& Mark, R.F. (1997) Conduction and synaptic transmission in the optic nerve and the superior colliculus during development of the retinocollicular projection in the wallaby (Macropus eugenii). J. Comp. Neurol., 380, 472-484.

Galli, L. \& Maffei, L. (1988) Spontaneous impulse activity of rat retinal ganglion cells in prenatal life. Science, 242, 90-91.

Grubb, M.S., Rossi, F.M., Changeux, J.P. \& Thompson, I.D. (2003) Abnormal functional organization in the dorsal lateral geniculate nucleus of mice lacking the beta 2 subunit of the nicotinic acetylcholine receptor. Neuron, $\mathbf{4 0}$, $1161-1172$.

Huberman, A.D., Stellwagen, D. \& Chapman, B. (2002) Decoupling eyespecific segregation from lamination in the lateral geniculate nucleus. J. Neurosci., 22, 9419-9429.

Huberman, A.D., Wang, G.Y., Liets, L.C., Collins, O.A., Chapman, B. \& Chalupa, L.M. (2003) Eye-specific retinogeniculate segregation independent of normal neuronal activity. Science, 300, 994-998.

Isa, T., Endo, T. \& Saito, Y. (1998) The visuo-motor pathway in the local circuit of the rat superior colliculus. J. Neurosci., 18, 8496-8504.

Itaya, S.K., Fortin, S. \& Molotchnikoff, S. (1995) Evolution of spontaneous activity in the developing rat superior colliculus. Can. J. Physiol. Pharmacol., 73, 1372-1377.

Juttner, R., Henneberger, C., Grantyn, R. \& Rothe, T. (2001) Early onset of glutamatergic and GABAergic synaptic activity in the visual layers of the rodent superior colliculus. Int. J. Dev. Neurosci., 19, 255-261.

Kirov, S.A., Sorra, K.E. \& Harris, K.M. (1999) Slices have more synapses than perfusion-fixed hippocampus from both young and mature rats. J. Neurosci., 19, 2876-2886.

Lim, C. (1997) Optic nerve conduction and the developmental onset of synaptic transmission in the mammalian superior colliculus. PhD Thesis. Australian National University, Australia.

Lim, C.H. \& Ho, S.M. (1997) Early detection of optic nerve-evoked response in the superior colliculus of the neonatal rat. Neurosci. Lett., 235, 141-144.

Lim, C.H. \& Ho, S.M. (1998) GABAergic modulation of axonal conduction in the developing rat retinotectal pathway. Brain Res. Dev. Brain Res., 108, 299-302.

Mark, R.F., Freeman, T.C., Ding, Y. \& Marotte, L.R. (1993) Two stages in the development of a mammalian retinocollicular projection. Neuroreport, $\mathbf{5}$, $117-120$.

Marotte, L.R., Vidovic, M., Wheeler, E. \& Jhaveri, S. (2004) Brain-derived neurotrophic factor is expressed in a gradient in the superior colliculus during development of the retinocollicular projection. Eur. J. Neurosci., 20, 843-847.

McLaughlin, T. \& O’Leary, D.D. (2005) Molecular gradients and development of retinotopic maps. Annu. Rev. Neurosci., 28, 327-355.

McLaughlin, T., Torborg, C.L., Feller, M.B. \& O’Leary, D.D. (2003) Retinotopic map refinement requires spontaneous retinal waves during a brief critical period of development. Neuron, 40, 1147-1160.

Molotchnikoff, S. \& Itaya, S.K. (1993) Functional development of the neonatal rat retinotectal pathway. Brain Res. Dev. Brain Res., 72, 300-304.

Mooney, R., Penn, A.A., Gallego, R. \& Shatz, C.J. (1996) Thalamic relay of spontaneous retinal activity prior to vision. Neuron, 17, 863-874.

Mrsic-Flogel, T.D., Hofer, S.B., Creutzfeldt, C., Cloez-Tayarani, I., Changeux, J.P., Bonhoeffer, T. \& Hubener, M. (2005) Altered map of visual space in the superior colliculus of mice lacking early retinal waves. J. Neurosci., 25, 6921-6928.

Penn, A.A., Riquelme, P.A., Feller, M.B. \& Shatz, C.J. (1998) Competition in retinogeniculate patterning driven by spontaneous activity. Science, 279, 2108-2112. 
Petrak, L.J., Harris, K.M. \& Kirov, S.A. (2005) Synaptogenesis on mature hippocampal dendrites occurs via filopodia and immature spines during blocked synaptic transmission. J. Comp. Neurol., 484, 183-190.

Plummer, K.L. \& Behan, M. (1993) Development of corticotectal synaptic terminals in the cat: a quantitative electron microscopic analysis. J. Comp. Neurol., 338, 458-474.

Reece, L.J. \& Lim, C.H. (1998) Onset of optic nerve conduction and synaptic potentials in superior colliculus of fetal rats studied in vitro. Brain Res. Dev. Brain Res., 106, 25-38.

Sachs, G.M., Jacobson, M. \& Caviness, V.S. Jr (1986) Postnatal changes in arborization patterns of murine retinocollicular axons. J. Comp. Neurol., 246, 395-408.

Sakatani, K., Hassan, A.Z. \& Ching, W. (1991) Age-dependent extrasynaptic modulation of axonal conduction by exogenous and endogenous GABA in the rat optic nerve. Exp. Neurol., 114, 307-314.

Sheng, X.M., Marotte, L.R. \& Mark, R.F. (1990) Development of connections to and from the visual cortex in the wallaby (Macropus eugenii). J. Comp. Neurol., 300, 196-210.
Shi, J., Aamodt, S.M. \& Constantine-Paton, M. (1997) Temporal correlations between functional and molecular changes in NMDA receptors and GABA neurotransmission in the superior colliculus. J. Neurosci., 17, 6264-6276.

Simon, D.K. \& O'Leary, D.D. (1992) Development of topographic order in the mammalian retinocollicular projection. J. Neurosci., 12, 1212-1232.

Simon, D.K., Prusky, G.T., O’Leary, D.D. \& Constantine-Paton, M. (1992) $N$-methyl-D-aspartate receptor antagonists disrupt the formation of a mammalian neural map. Proc. Natl Acad. Sci. USA, 89, 10593-10597.

Skoff, R.P. \& Hamburger, V. (1974) Fine structure of dendritic and axonal growth cones in embryonic chick spinal cord. J. Comp. Neurol., 153, 107-147.

Spira, A.W. \& Marotte, L.R. (1989) Histological and electron microscopic milestones in the development of the retina of a marsupial wallaby, Macropus eugenii. Anat. Embryol. (Berl.), 179, 571-585.

Warton, S.S. \& McCart, R. (1989) Synaptogenesis in the stratum griseum superficiale of the rat superior colliculus. Synapse, 3, 136-148.

Warton, S.S., Perouansky, M. \& Grantyn, R. (1990) Development of GABAergic synaptic connections in vivo and in cultures from the rat superior colliculus. Brain Res. Dev. Brain Res., 52, 95-111. 